\title{
A NOTE ON MODULES OVER A COMMUTATIVE REGULAR RING
}

\author{
MARK L. TEPLY
}

\begin{abstract}
An example is given of a commutative, von Neumann regular ring $R$, which has a module $A$ satisf ying the following conditions: (1) $T(A)=\{a \in A \mid(0: a)$ is an essential ideal of $R\}$ is a cyclic $R$-module; (2) $A / T(A)$ is a cyclic $R$-module; and (3) $T(A)$ is not a direct summand of $A$. This answers in the negative a question raised by $\mathrm{R}$. S. Pierce.
\end{abstract}

In Pierce's memoir [2], a module $M$ over a commutative regular ring $R$ is called torsion if $\operatorname{Hom}_{R}(M, E(R))=0$, where $E(R)$ denotes the injective hull of $R$. This definition for torsion modules over a commutative regular ring coincides with several other concepts of torsion for modules over more general rings (see [1]). The following question has been asked by Pierce [2, p. 109]: "If the torsion submodule $T(A)$ is finitely generated and $A / T(A)$ is a finite direct sum of cyclic modules, does it follow that $T(A)$ is a direct summand of $A$ ?" The purpose of this note is to answer this question in the negative by giving an example of a module $A$ such that $T(A)$ and $A / T(A)$ are cyclic, but $T(A)$ is not a summand of $A$.

Let $B$ be a non-Artinian Boolean ring with identity 1 . For each positive integer $n$, let $B^{(n)} \cong B$. Let $P=\prod_{n=1}^{\infty} B^{(n)}$, and define elements $e$ and $f$ of $P$ as follows:

$$
e=(1,1,1, \cdots)
$$

and

$$
\begin{array}{rlrl}
f=\left(a_{1}, a_{2}, a_{3}, \cdots\right), \quad \text { where } a_{n} & =1 \quad & \text { if } n=2 k, \quad k=1,2, \cdots, \\
& =1 \quad \text { if } n=3^{s}, \quad s=1,2, \cdots, \\
& =0 \quad \text { otherwise. }
\end{array}
$$

Let $R$ be the subring of $P$ generated by $e$ and $\oplus \sum_{n=1}^{\infty} B^{(n)}$. Since $B$ is not Artinian, there exists an essential ideal $I$ of $B$. Let $N_{0}=\bigoplus \sum_{n=1}^{\infty} I^{(n)}$ and $N_{1}=\oplus \sum_{n=1}^{\infty} I^{(2 n)}$, where $I^{(n)} \cong I$ for each integer $n$. Let $N$ be the $R$-submodule of $\left(R e / N_{0}\right) \oplus\left(R f / N_{1}\right)$ generated by all elements of the form

Received by the editors November 16, 1970.

AMS 1970 subject classifications. Primary 13C10, 16A30; Secondary $18 \mathrm{E} 40$.

Key words and phrases. Regular ring, torsion submodule, Boolean ring, direct summand, cyclic module.

Copyright () 1971, American Mathematical Soclety 


$$
\left(\left(0, v_{2}, 0, v_{4}, 0, v_{6}, \cdots\right)+N_{0},\left(0, v_{2}, 0, v_{4}, 0, v_{6}, \cdots\right)+N_{1}\right) .
$$

Define $A=\left(\left(\operatorname{Re} / N_{0}\right) \oplus\left(R f / N_{1}\right)\right) / N$.

Since a commutative regular ring $R$ has zero singular ideal, it is well known (see [1]) that an element $x$ of an $R$-module $X$ is in $T(X)$ if and only if $(0: x)$ is an essential ideal of $R$. From this fact and the definition of $N$, it can be verified that $T(A)$ is generated by the element $\left(e+N_{0},(0,0,0, \cdots)+N_{1}\right)+N$.

Since $T\left(R e / N_{0}\right)=R e / N_{0}$, it follows from the closure properties (see [1] or [2]) of torsion classes that there is a naturally induced epimorphism from

$$
R f / N_{1} \cong\left(\left(R e / N_{0}\right) \oplus\left(R f / N_{1}\right)\right) /\left(R e / N_{0}\right)
$$

onto $A / T(A)$. Therefore $A / T(A)$, being the epimorphic image of a cyclic module, is cyclic.

Let $R y \subseteq A$ such that $T(R y)=0$, and write

$$
y=\left(\left(t_{1}, t_{2}, t_{3}, \cdots\right)+N_{0},\left(b_{1}, b_{2}, b_{3}, \cdots\right)+N_{1}\right)+N .
$$

Now let $h_{k}=\left(c_{1}, c_{2}, c_{3}, \cdots\right)$, where $c_{k}=1$ and $c_{i}=0$ for all $i \neq k$. Thus $h_{5^{i} y}=\left(\left(0,0, \cdots, 0, t_{5^{i}}, 0, \cdots\right)+N_{0},(0,0, \cdots)+N_{1}\right)+N \in T(A)$ for all $i=1,2, \cdots$. Since $T(R y)=0$, it follows that $t_{5 i} \in I^{\left(5^{i}\right)}$ for all $i=1,2, \cdots$. Hence there exists a positive integer $k$ such that $t_{i}$ $\in I^{(i)}$ for all $i \geqq k$. Then

$$
\begin{aligned}
h_{2 i} y=\left(\left(0,0, \cdots, 0, t_{2 i},\right.\right. & 0, \cdots) \\
& \left.+N_{0},\left(0,0, \cdots, 0, b_{2 i}, 0, \cdots\right)+N_{1}\right)+N
\end{aligned}
$$

is an element of $T(A)$; hence $b_{2 i} \in I^{(2 i)}$ for all $2 i \geqq k$. Thus there exists a positive integer $s \geqq k$ such that $b_{i} \in I^{(i)}$ for all $i \geqq s$. It is now easy to verify that

$$
\left((0,0, \cdots)+N_{0}, h_{3^{\circ}}+N_{1}\right)+N \notin T(A) \oplus R y .
$$

If $T(A)$ were a direct summand of $A$, then by the fact that $A / T(A)$ is cyclic, there would exist a cyclic module $R y \subseteq A$ such that $T(R y)$ $=0$ and $A=T(A) \oplus R y$. By the preceding paragraph, such a module $R y$ cannot exist; hence $T(A)$ cannot be a direct summand of $A$.

\section{REFERENCES}

1. J. S. Alin and S. E. Dickson, Goldie's torsion theory and its derived functor, Pacific J. Math. 24 (1968), 195-203. MR 37 \#2834.

2. R. S. Pierce. Modules oier commutative regular rings, Mem. Amer. Math. Soc. No. 70 (1967). MR 36 \#151.

University of Florida, Gainesville, Florida 32601 